\title{
Golgi-Disturbing Agents Lead to the Elimination of Intracellular Toxoplasma gondii
}

\author{
C. S. Carvalho, G. R. Figueiredo and E. J. T. de Melo* \\ Laboratório de Biologia Celular, Centro de Biociências e Biotecnologia, Universidade Estadual do Norte Fluminense
}

\begin{abstract}
The Golgi apparatus is responsible for the genesis of secretory organelles of Toxoplasma gondii and lipid traffic to the vacuole. This study used anti-Golgi agents to demonstrate the importance of Golgi in Toxoplasma development. Monensin, Brefeldin A, Retinoic Acid and Okadaic Acid reduced the infection, leading to parasite elimination. Mon, BFA and RA affected secretory organelles and the Golgi Complex of the parasites, with faster parasite elimination in the presence of Monensin; in addition, the vesicular transit of host cell C6-NBD-ceramide metabolites was interrupted, but the GC of host cells was preserved. Our results suggest that several targets in the secretory pathway are affected in the intracellular Toxoplasma rather than in the host cells, resulting in interruption of parasite development and its elimination from the intracellular medium.
\end{abstract}

Keywords: Anti-Golgi agents, lipid traffic, Toxoplasma gondii.

\section{INTRODUCTION}

Toxoplasma gondii is a ubiquitous parasite that infects virtually all nucleated cells of vertebrates [1]. The success of intracellular parasitism is due to the differentiated niche provided by the parasite. This niche is formed by the tachyzoite - the infective form of T. gondii - and the parasitophorous vacuole (PV), in which the parasite develops and multiplies until lysis of the host cell [2]. Following intracellular infection, host cell organelles, such as mitochondria and endoplasmic reticulum, change their cytoplasmatic distribution, and join the parasitophorous vacuole membrane [3-5]. These events are vital to parasite survival. Moreover, the PV-containing tachyzoites also localize close to the host cell nucleus and the Golgi complex (GC).

The GC is fundamental to cellular homeostasis for it receives newly synthesized molecules from the endoplasmic reticulum; and it covalently modifies and distributes them to the plasma membrane, lysosomes and secretory vesicles. Furthermore, the GC is closely tied to membrane mechanisms and metabolite traffic, where it undergoes continuous changes and remodeling, with its dynamics decisive for vesicle transport and cell survival [6]. In $T$. gondii, the presence of a single Golgi complex was confirmed recently by Pelletier et al. [7]. The GC in $T$. gondii has been shown to be essential for the formation of three specialized secretory organelles: rhoptries, micronemes and dense granules [8], which are essential for invasion and parasite intracellular development. Moreover, during parasite development, intravacuolar tachyzoites take up essential metabolites from the GC of host cells [9-13].

Based on this, pharmacological agents have proven useful for gaining insights into the structure and function of

*Address correspondence to this author at the Avenida Alberto Lamego 2000, Campos dos Goytacazes, CEP 28015-620, Rio de Janeiro, Brazil; Fax: +55 (22) 27261500; E-mail: ejtm1202@oi.com.br the Golgi complex. The perturbation of Golgi action is recognized by drug effects, such as blocking secretion, altering vesicular morphology, loss of cisternal stacks or inhibition of vesicular transport [14]. Here, we used several classic pharmacological Golgi-disturbing agents to gain new insights about the importance of this organelle for intracellular Toxoplasma gondii development.

\section{MATERIALS AND METHODOLOGY}

\section{Parasites}

Tachyzoites from the virulent RH strain of Toxoplasma gondii were maintained by routine intraperitoneal passage in Swiss mice. To carry out in vitro infection, tachyzoites were harvested from the peritoneal cavity with phosphate-buffered saline solution (PBS) at pH 7.2, 48 hours after infection. The ascitic fluid obtained from infected mice was centrifuged at $260 \mathrm{~g}$ for 10 minutes at room temperature. The pellet obtained was washed once with PBS, pH 7.2, and suspended to a density of $10^{7}$ parasites $/ \mathrm{ml}$ in 199 medium with $5 \%$ fetal bovine serum (SFB).

\section{Host Cell}

Vero cells (kidney fibroblast of African green monkey) were maintained in Falcon plastic flasks using 199 medium with 5\% SFB. At confluent monolayer, cells were washed with PBS and harvested using EDTA-trypsin solution. Approximately $4 \times 10^{4}$ Vero cells were seeded in 24 -well plates that contained a round sterile coverslip, or $4 \times 10^{6}$ cells were seeded in culture flasks $\left(75 \mathrm{~cm}^{2}\right)$ and maintained overnight at $37^{\circ} \mathrm{C}$ in $5 \% \mathrm{CO}_{2}$.

\section{Host Cell-Parasite Interaction}

Parasite suspension was prepared as described above and used within $1 \mathrm{~h}$ after peritoneal harvest. The Vero cell cultures were infected using a 5:1 parasite-host cell ratio. The cultures were incubated for $1 \mathrm{~h}$ at $37^{\circ} \mathrm{C}$ and washed 
with PBS to remove non-adherent parasites. After that, they were incubated in the Vero cells for $24 \mathrm{~h}$ at $37{ }^{\circ} \mathrm{C}$, in $5 \%$ $\mathrm{CO}_{2}$, and then infected cells were used in all experiments, as in the following description.

\section{Anti-Golgi Agents}

The drugs were used in different concentrations according to the expected effect on the Golgi complex, as previously reviewed by Dinter and Berger [13]. The following drugs were added to infected host cells for 1 to $24 \mathrm{~h}$ :

- Monensin was obtained from Sigma Chemical Co. It was dissolved in methanol at a concentration of 2 $\mathrm{mg} / \mathrm{mL}$ and stored at $-70{ }^{\circ} \mathrm{C}$. For the experiments, Monensin was added to the infected cultures at a concentration of $10 \mu \mathrm{M}$, at $37^{\circ} \mathrm{C}$, in $5 \% \mathrm{CO}_{2}$.

- $\quad$ Brefeldin A was also obtained from Sigma Chemical Co. It was dissolved in dimethyl sulfoxide (DMSO) at a concentration of $10 \mathrm{mg} / \mathrm{mL}$, stored at $-70{ }^{\circ} \mathrm{C}$. The experiments were carried out with BFA $3.5 \mu \mathrm{M}$, in infected Vero cells as described above.

- $\quad$ Retinoic acid was obtained from Merck Chemical Co. and it was dissolved in dimethyl sulfoxide (DMSO) at a stock concentration of $2 \mathrm{mg} / \mathrm{mL}$, stored at $-70{ }^{\circ} \mathrm{C}$, and used at a final concentration of $10 \mu \mathrm{M}$.

- $\quad$ Okadaic acid was obtained from Sigma Chemical Co. It was dissolved in dimethyl sulfoxide (DMSO) at a concentration of $50 \mathrm{mg} / \mathrm{mL}$, stored at $-70{ }^{\circ} \mathrm{C}$, and it was used at a concentration of $0.5 \mu \mathrm{M}$.

\section{Morphological Study and Measure of Infection}

Following treatment (or not) with the above compounds, the infected cultures were rinsed with PBS buffer, fixed with Bowin's solution, and stained with Giemsa stain for $2 \mathrm{~h}$ at room temperature. After that the cultures were dehydrated in an acetone-xilol series. The cells were observed using an AXIOPLAN microscope equipped with a $63 \mathrm{X}$ objective. Images were obtained using analySIS (Soft imaging system) software. Infection was assessed by counts of monolayers on each three or four replicate slides per point or treatment. The percentages of infected cells and intracellular parasites were determined by examination of at least 600 cells in the same monolayer, at a magnification of X 400 [15]. The results shown in the graphs of each incubation time refer to independent experiments. Each drug incubation was compared with the untreated control. By counting these cells, the mean number of intracellular parasites was measured. Results are expressed as averages \pm standard error. The results were plotted using Excel software.

\section{Statistical Analysis}

The statistical analysis was carried out using the Student T-test with the level of significance SRT at $p<0.05$. The data shown are representative of four experiments in triplicate.

\section{Fluorescent Lipid Traffic and Brefeldin Observations}

Brefeldin A-BODIPY and 6-((n-(7-nitrobenz-2-oxa-1,3diazol-4-yl)amino) hexanoyl) sphingosine) ( $\mathrm{C}_{6}$-NBD-cera- mide) were obtained from Molecular Probes Inc. (Eugene, OR). Brefeldin A-BODIPY, at a concentration of $3.5 \mu \mathrm{M}$ in 199 medium, was added to cultures for 30 minutes at $37^{\circ} \mathrm{C}$, $5 \% \mathrm{CO}_{2}$, to observe Golgi complex and drug localization in the intravacuolar parasites. To observe the lipid traffic in the presence of anti-Golgi agents, Monensin $(10 \mu \mathrm{M})$ or BFA $(3.5 \mu \mathrm{M})$ were added to infected cultures for 1 or 3 hours, respectively. $\mathrm{C}_{6}$-NBD-Cer, dissolved in dimethyl sulfoxide at a concentration of $1.4 \mathrm{mg} / \mathrm{mL}$ in 199 medium, was incubated in the cultures in the presence of drugs for $5 \mathrm{~h}$ at $37{ }^{\circ} \mathrm{C}, 5 \% \mathrm{CO}_{2}$. The specimens were examined using a Zeiss Confocal Laser Scanning Microscope (CLSM), with a 488 and $543 \mathrm{~nm}$ argon laser.

\section{Ultrastructural Aspects}

Vero cells were plated in culture flasks $\left(75 \mathrm{~cm}^{2}\right)$, cultivated as described above, and allowed to interact with the parasites. After drug incubation, the cultures were washed with PBS and fixed for $1 \mathrm{~h}$ in solution containing $1 \%$ glutaraldehyde, $4 \%$ formaldehyde, $5 \mathrm{mM} \mathrm{CaCl}_{2}$, and $5 \%$

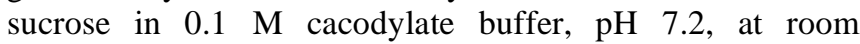
temperature. The samples were washed with solution containing $0.1 \mathrm{M}$ cacodylate buffer and 5\% sucrose, and post fixed for $1 \mathrm{~h}$ in a solution containing $1 \% \mathrm{OSO}_{4}, 0.8 \%$ potassium ferrocyanide, and $5 \mathrm{mM} \mathrm{CaCl} 2$ in $0.1 \mathrm{M}$ cacodylate buffer, $\mathrm{pH}$ 7.2. The cells were then washed with $0.1 \mathrm{M}$ cacodylate buffer, dehydrated in an acetone ascendant series, and embedded in Epon. Thin sections were stained with aqueous solution containing $2 \%$ uranyl acetate and $0.3 \%$ lead citrate. The cells were observed using a Zeiss 900 transmission electron microscope, at $80 \mathrm{kV}$.

\section{RESULTS}

Vero cells were cultivated as previously described and infected with tachyzoites of $T$. gondii for $24 \mathrm{~h}$. During this time, a high load of intracellular parasites was observed in the host cells. The Golgi-disrupting pharmacological agents were incubated with these cultures for different periods. Drug concentrations were based on Dinter and Berger [14]. Monensin $(10 \mu \mathrm{M})$, Brefeldin A $(3.5 \mu \mathrm{M})$, Retinoic acid $(10$ $\mu \mathrm{M})$ and Okadaic acid $(0.5 \mu \mathrm{M})$ were incubated in cultures for 1 to 24 hours. We accomplished viability assays by quantifying the cells in the culture and relating them to the control (untreated cells), and by an exclusion viability test using Trypan blue. Non-toxic effects were assumed when the number of total cells in the treated culture was similar to those counted in the untreated cultures, and cells were negative for Trypan blue. However, toxic effects on the host cell were observed at different times for each drug. Mon and BFA did not induce cytotoxic effects until $12 \mathrm{~h}$ of incubation. RA was toxic only after $24 \mathrm{~h}$ of incubation, while OA was highly toxic after $7 \mathrm{~h}$ of incubation. Thus we set up some points in the graphs to demonstrate drug action on intracellular parasites.

In infected cultures, Monensin (Mon) and Brefeldin A (BFA) showed anti-parasitic action as demonstrated in Fig. (1). Both drugs caused a significant decrease in the infection (Figs. 1a,c) and in the mean number of intracellular tachyzoites of $T$. gondii (Figs. 1b,d). However, the infection decreased by about $70 \%$ after $1 \mathrm{~h}$ of incubation with Mon (Figs. 1a,b) while a decrease of $50 \%$ took place after $5 \mathrm{~h}$ of 



Intracelullar parasites

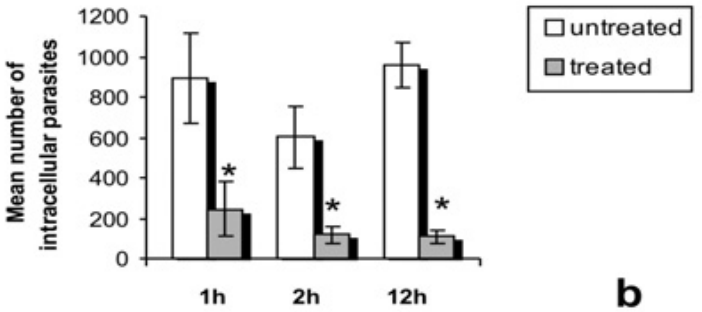

Infected Vero cells treated with Brefeldin A
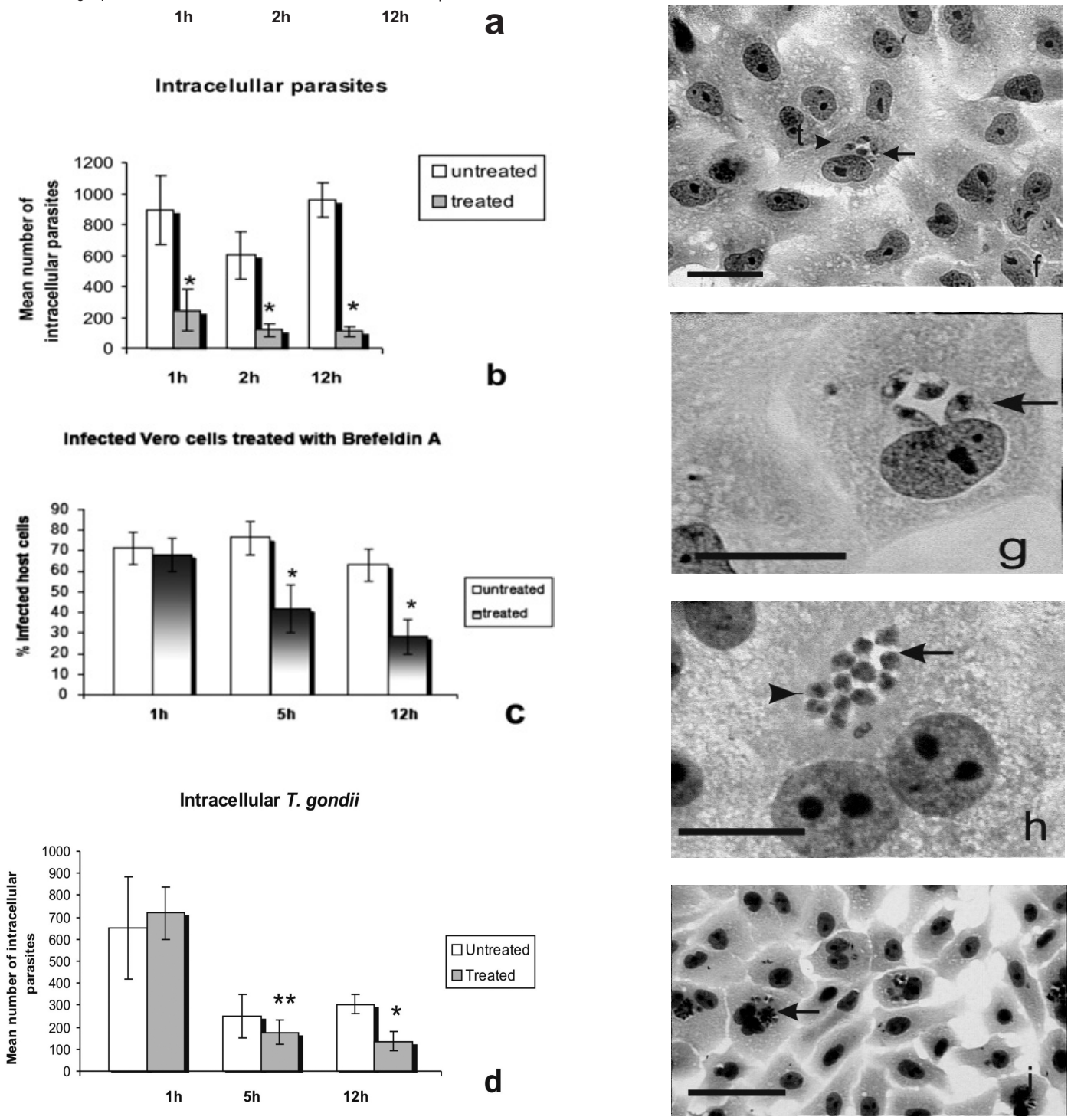

Fig. (1). Infected Vero cells treated with Monensin (Mon) and Brefeldin A (BFA) decreased percentage of infection (a, c) and mean number of intracellular tachyzoites (b, d), respectively. Morphological aspects of untreated infected culture (e) and infected culture treated with Mon 1h (f, g) or BFA 5h (h, i) were observed. Both of drugs caused wide elimination of intracellular tachyzoites (f, i). Remains parasites within parasitophorous vacuole (PV) were drastically disorganized (g, h). Bars: e, f, $\mathrm{i}=25 \mu \mathrm{m}$; g, $\mathrm{h}=50 \mu \mathrm{m}$. Asterisks indicative significant differences as determined by Student's T-test: $* \mathrm{P}<0.0001 ; * * \mathrm{P}=0.014$.

BFA incubation (Fig. 1c). In this time a few intravacuolar parasites were eliminated (Fig. 1d). A significant reduction in infection and intravacuolar parasites was only seen after $12 \mathrm{~h}$ of BFA incubation (Figs. 1c,d). These drugs were also incubated with extracellular parasites; Mon for 10 to $30 \mathrm{~min}$ and BFA for $10 \mathrm{~min}$. Invasion and parasite development were determined after incubation with both drugs. Mon and
BFA inhibited parasite invasion by $60 \%$ and $40 \%$, respectively. In the case of Mon, treated extracellular parasites were not able to develop after $24 \mathrm{~h}$ of infection (data not shown).

In addition to the effect of Mon and BFA on infection reduction, the morphological aspects of infected cultures 
treated with both compounds were analyzed. Normal intravacuolar tachyzoites had a crescent shape (Fig. 1e). After Mon treatment a significant reduction in the number of parasites was observed, however altered morphologically tachyzoites could still be seen. This was also observed

Infected Vero cells treated with Retinoic acid

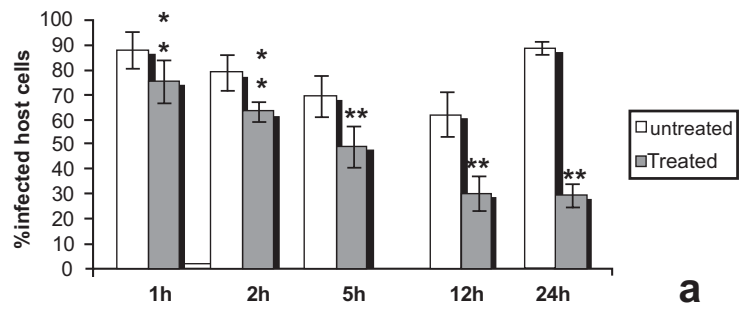

Intracellular Tachyzoites

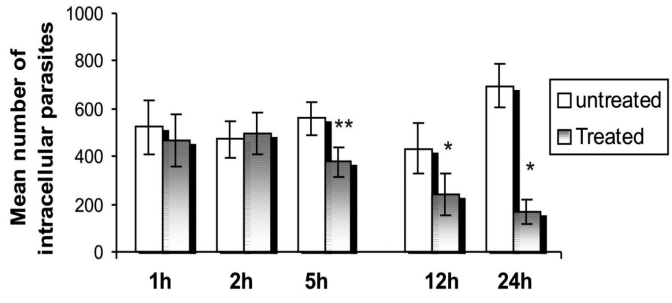

b

Infected Vero cells treated with Okadaic acid

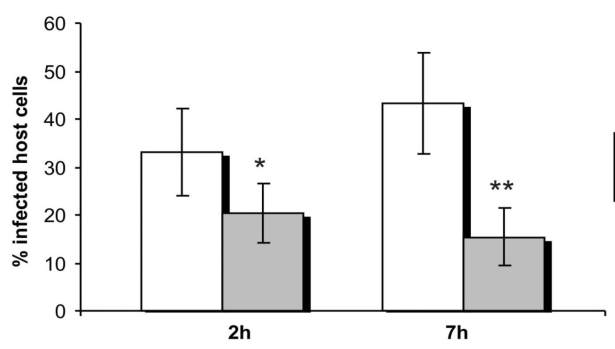

$\square$ untreated

Intracellular tachyzoites

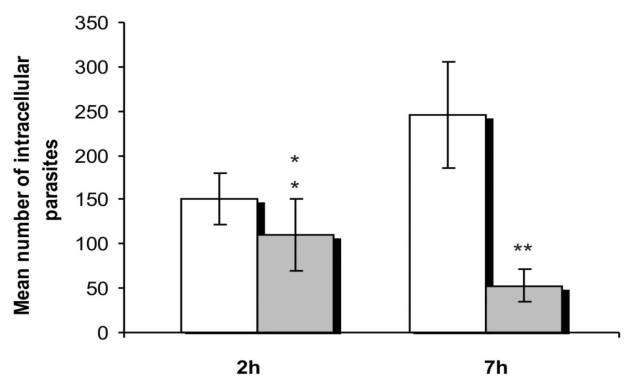

C

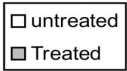

d during BFA treatment for $5 \mathrm{~h}$ (Fig. 1h) and $12 \mathrm{~h}$ (Fig. 1i), although some integral tachyzoites remained inside the PV after BFA incubation (Fig. 1i). Nevertheless, cells were spread on the substrate during incubation with both drugs.
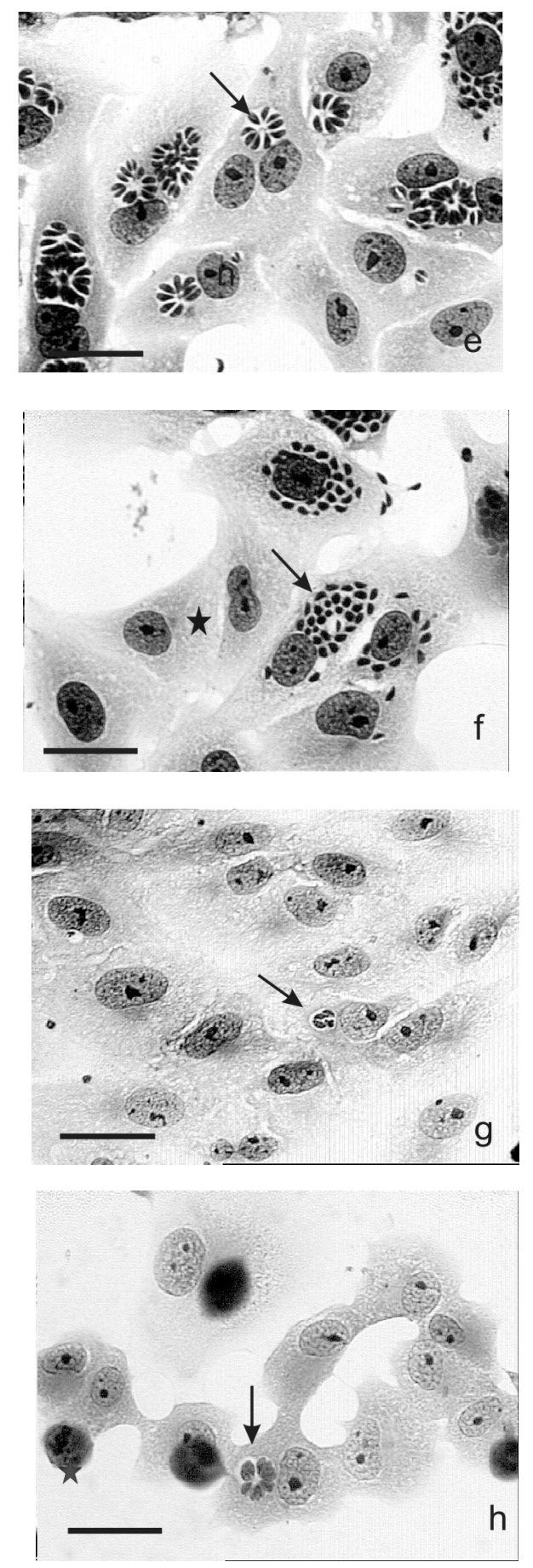

Fig. (2). Infected Vero cells treated with retinoic (RA) and okadaic acid (OA). Percentage of infection (a, c) and mean number of intracellular tachyzoites $(\mathbf{b}, \mathbf{d})$ decreased after drugs incubation. Control cultures had parasites multiplying (e). RA incubated for $5 \mathrm{~h}(\mathbf{f})$ led to a decrease in PV-containing parasites, but only after $12 \mathrm{~h}$ of treatment $(\mathbf{g})$ wide reduction in infection and drastic disorganization in parasites were noted (g). OA initially reduced infection after $2 \mathrm{~h}$ of treatment (c) but only after $7 \mathrm{~h}(\mathbf{h})$ the drastic reduction occurred. Mean number of intravacuolar parasites also was reduced, mostly after $7 \mathrm{~h}$ of drug incubation, but toxic effects (cell death) on host cells (h, star) appeared. Arrows $=$ parasitophorous vacuole; $\mathrm{t}=$ tachyzoites; $\mathrm{n}=$ nucleus. Bar $=25 \mu \mathrm{m}$. Asterisks indicative significant differences as determined by Student's T-test: $* \mathrm{P}<0.0004 ; * * \mathrm{P}<0.0001 ; * * * \mathrm{P}<0.003$. 
Retinoic and okadaic acid treatment also induced a decrease in the number of infected cells and number of intracellular parasites (Fig. 2). Unlike Mon, Retinoic acid (RA) reduced infection by $50 \%$ after only $5 \mathrm{~h}$ of incubation (Fig. 2a), and some of the normal intravacuolar parasites persisted until $24 \mathrm{~h}$ of treatment (Figs. 2b, 2f). In the same way, the treatment of infected cultures with okadaic acid (OA) resulted in a decrease in infection (Fig. 2c) and in the number of intravacuolar tachyzoites (Fig. 2d), after $2 \mathrm{~h}$ of incubation. Furthermore, drastic effects were observed after $7 \mathrm{~h}$ of incubation. From this time, OA induced cytotoxic effects in the host cells, such as loss of cytoplasm volume and adhesiveness, nucleus condensation and death (Fig. 2h). However, persistent intravacuolar parasites could still be seen in some cells (Fig. $2 \mathbf{h}$ ).

The parasites remaining in the culture following treatment with the drugs showed normal or altered morphology. We tested whether these persistent parasites resume multiplication when the anti-Golgi agents were removed. Thus, infected cells were treated, and after that the drugs were removed and the cultures incubated with fresh medium for $24 \mathrm{~h}$. The cultures were analyzed, and the infection and number of intracellular parasites were determined as described earlier. The infected cells treated with Mon did not recover from its effect on intravacuolar parasites. Nevertheless, after BFA and RA treatment, tachyzoites resumed multiplication. However, for these two drugs reversion occurred only after $7 \mathrm{~h}$ of incubation; longer incubation prevented return to multiplication. OA again caused irreversible cytotoxicity (data not shown).

As the different anti-Golgi agents have several targets, we decided to investigate possible synergistic effects between them. Mon plus BFA reduced $T$. gondii infection and eliminated parasites in the same way as Mon alone (Figs. 3a,b). The inclusion of retinoic acid in this combination (Mon+BFA+RA) also had no further effect on Mon
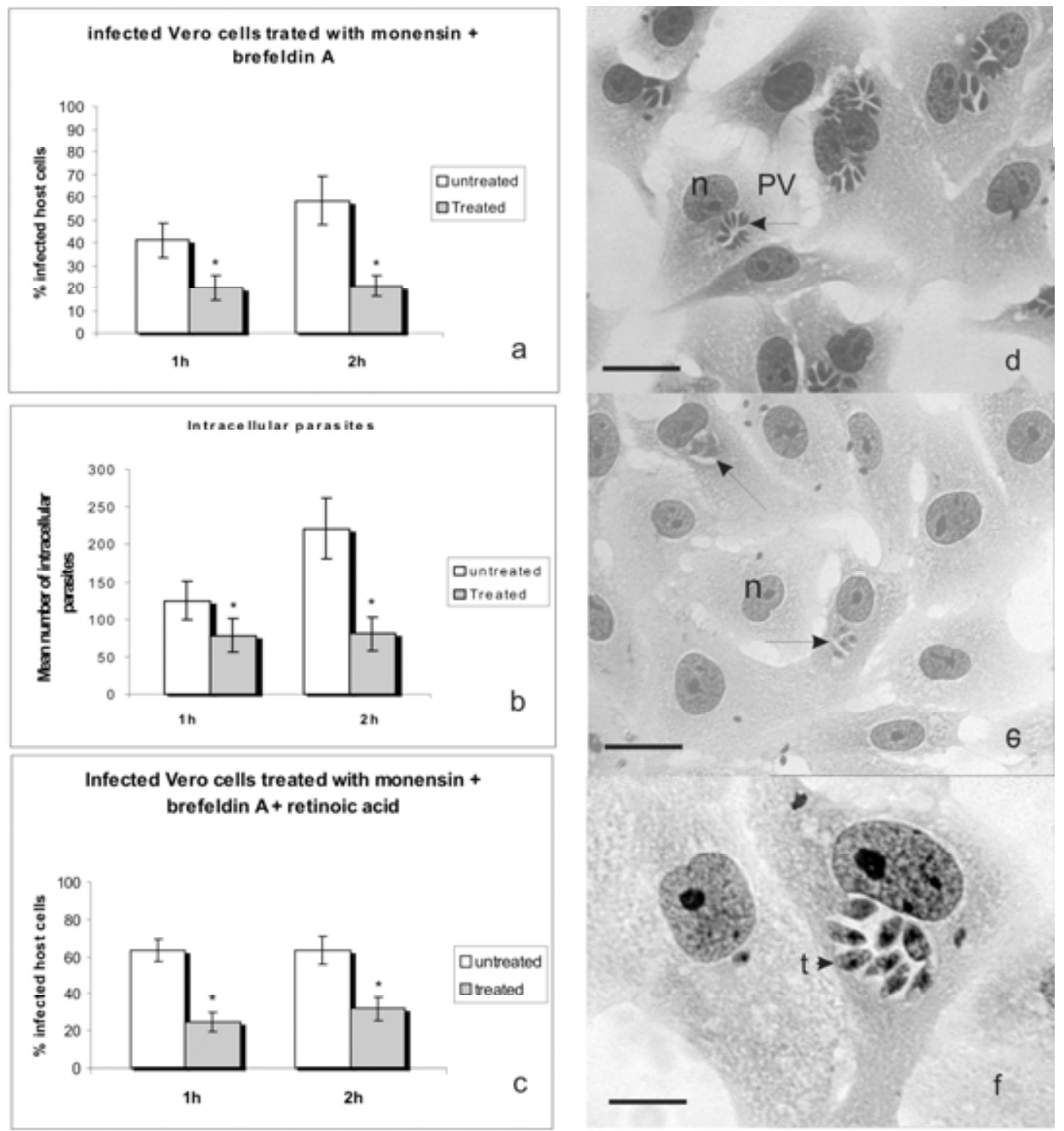

Fig. (3). Infected Vero cells were treated with combination of Monensin (Mon), Brefeldin A (BFA) and Retinoic Acid (RA). Mon + BFA (a, b) resulted in significant reduction of infection (a) and the mean number of intracellular tachyzoites (b, e). All associated drugs also reduced the infection (c) and induced drastic disorganization in the intravacuolar tachyzoites $(\mathbf{f})$. No toxic effect on the host cells was noted. Arrows $=$ parasitophorous vacuole; $\mathrm{n}=$ nucleus; $\mathrm{t}=$ tachyzoites. Bars: $\mathrm{d}, \mathrm{e}=25 \mu \mathrm{m} ; \mathrm{f}=50 \mu \mathrm{m}$. Asterisks indicative significant differences as determined by Student's T-test: $* \mathrm{P}<0.0001$. 

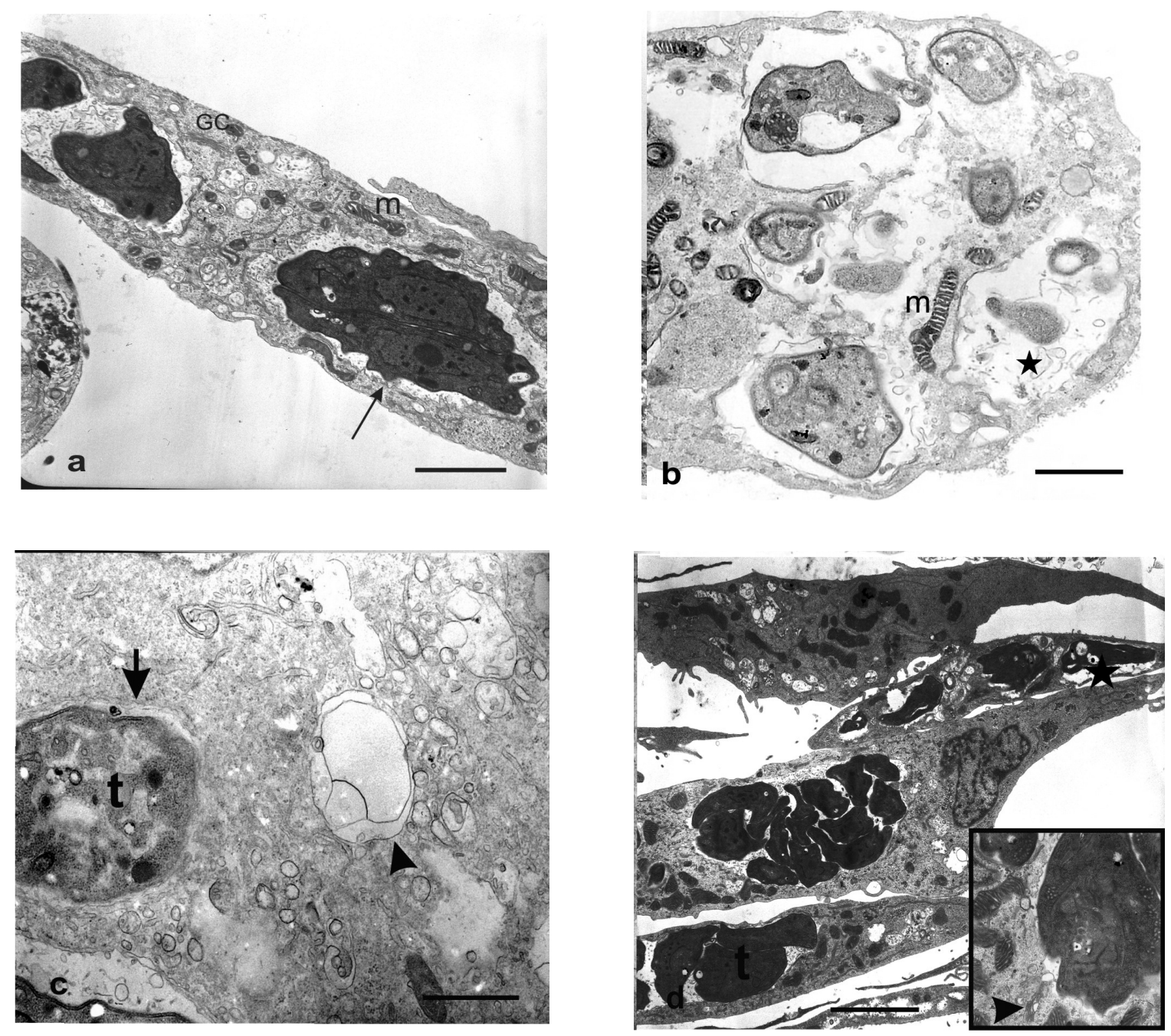

Fig. (4). Ultrastructural aspects of drugs treatment. In untreated cells intravacuolar parasites (arrow) with normal features were observed (a). Incubation of infected cultures with Monensin for $2 \mathrm{~h}(\mathbf{b})$ induced different stages of tachyzoites disorganization. Vesiculations in the Tachyzoites were drastically observed in Mon (b) and BFA (c). Retinoic acid (RA) incubated for 12h (d) caused little vesiculations in the parasites (d), but disorganized parasites also could be seen. Only BFA incubated for $5 \mathrm{~h}$ induced vesiculation of host cytoplasm organelles, mainly Golgi complex (c, arrowhead). $\mathrm{N}=$ nucleus; $\mathrm{m}=$ mitochondria; Golgi complex $(\mathrm{GC}) ; \mathrm{t}=$ tachyzoites. $\mathrm{Bars}=\mathrm{a}, \mathrm{b}, \mathrm{c}=5 \mu \mathrm{m} ; \mathrm{d}=10$ $\mu \mathrm{m}$.

action (Fig. 3c). Cytotoxic effects were not observed. However, OA treatment combined with any other drug had a strong cytotoxic effect (data not shown).

The ultrastructural effects of Monensin, Brefeldin and Retinoic acid on parasites and host cells were analyzed. Parasites in untreated cultures showed integral cytoplasm; rhoptries were visible (Fig. 4a). Host cell organelles, such as mitochondria and endoplasmic reticulum, were associated with the PV, and the Golgi complex was also seen in the host cells (Fig. 4a). During incubation with anti-Golgi agents, the endomembrane system of tachyzoites underwent drastic vesiculations (Figs. 4b-d). In the presence of Mon (1 h), BFA ( $2 \mathrm{~h})$ and RA (12 h), vesiculations could be seen in the parasite Golgi complex and secretory organelles, such as roptries and micronemes. However, this structural disorganization preferentially affected the intravacuolar parasites, as observed in the $2 \mathrm{~h}$ Mon treatment (Fig. 4b). Mon and RA did not change the ultrastructural aspect of the host cell Golgi complex (Figs. 4b and 4d), however during BFA incubation, the Golgi complex of host cells also underwent vesiculations (Fig. 4c).

Given the ultrastructural alterations observed during incubation with anti-Golgi agents, and that the Golgi complex has a central point in metabolic traffic [6], we analyzed the lipid traffic of the host cell using exogenous lipids $-\mathrm{C}_{6}$-NBD-ceramide, as demonstrated by Melo and De Souza [9]. These authors and others [30] showed that NBDceramide stains the GC where it is metabolized into two fluorescent metabolites, which are then transported to the plasma membrane and intravacuolar tachyzoites after $5 \mathrm{~h}$, as shown in Fig. (5a). When infected cultures were pre-treated with Mon for $1 \mathrm{~h}$ and then incubated for $5 \mathrm{~h}$ with $\mathrm{C}_{6}-\mathrm{NBD}$ ceramide, an intense fluorescent stain was observed in the perinuclear region (GC) of the host cell, but it was missing from intravacuolar parasites (Figs. 5a-d). When infected cells were incubated with fluorescent Brefeldin A-BODIPY $(3.5 \mu \mathrm{M})$ for $30 \mathrm{~min}$, an intense fluorescence pattern was observed in the host cell perinuclear region and in the 


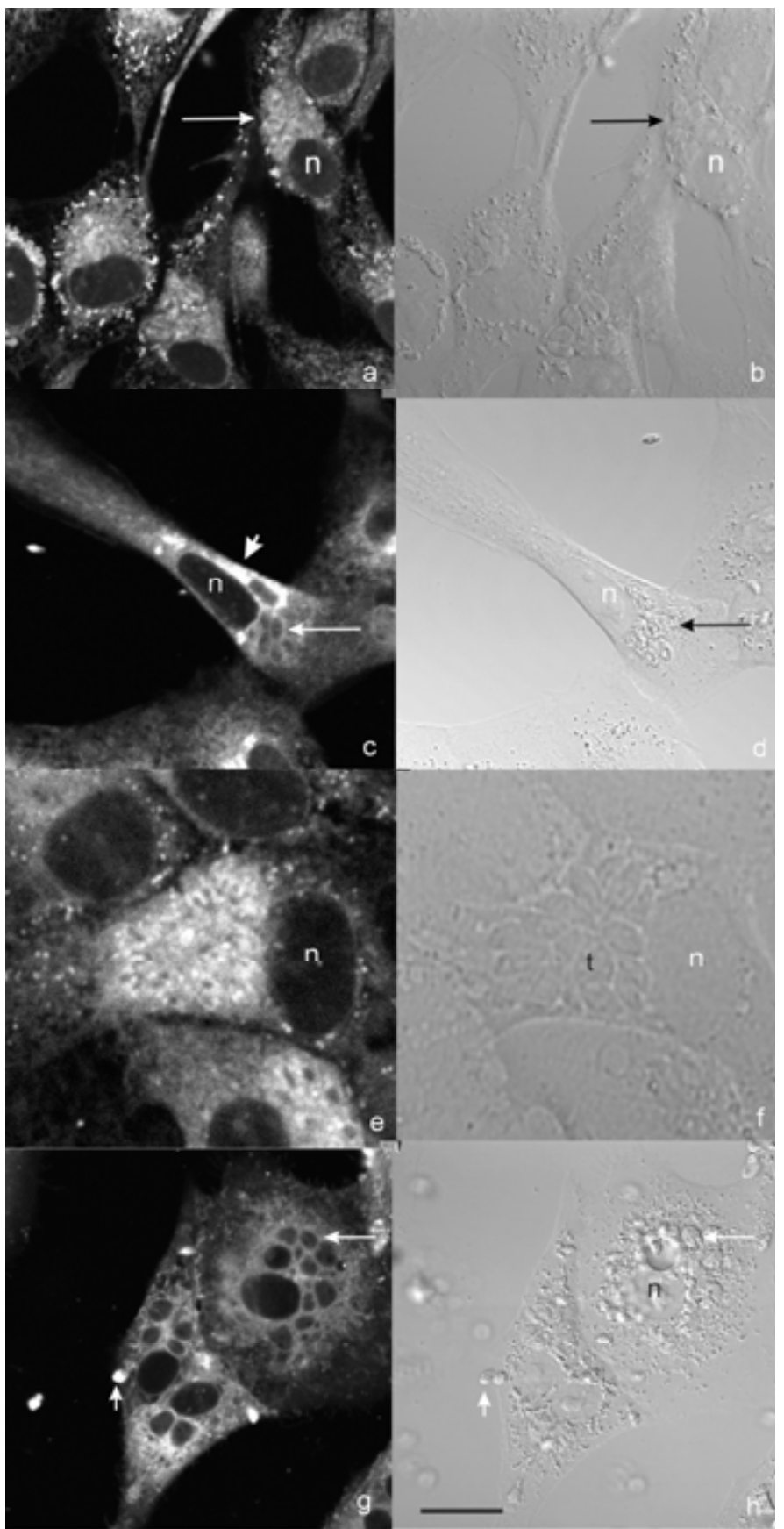

Fig. (5). $\mathrm{C}_{6}$-NBD-ceramide traffic in infected host cells $(\mathbf{a}, \mathbf{b})$ treated with Monensin for $1 \mathrm{~h}(\mathbf{c}, \mathbf{d})$, Brefeldin A for $30 \mathrm{~min}(\mathbf{e}, \mathbf{f})$ and $3 \mathrm{~h}(\mathbf{g}, \mathbf{h})$. Infected host cells incubated for $5 \mathrm{~h}$ with lipid-probe showed spread fluorescent spots into cytoplasm of host cells (a, b) and intravacuolar tachyzoites (a, b; arrow). When infected cultures were treated with Monensin for $1 \mathrm{~h}$ and after that incubated with lipid-probe (for 5h), an intense fluorescent stain (c, arrowhead) was close to the nucleus (n) but it was abolished within intravacuolar space (c, arrow) where remaining tachyzoites were morphologically disorganized (d, arrow). Brefeldin A-BODIPY - fluorescent probe - was incubated for 30 minutes and showed that intravacuolar parasites $(t)$ were intensely fluorescent $(\mathbf{e}, \mathbf{f})$. When infected cells were only treated with BFA and than incubated for 5h with lipid-probe, the host cell cytoplasm and extracellular parasites (g, arrowhead) presented fluorescent stains, but no stain was observed within PV space (g, arrow) where disorganized parasites were presented (h, arrow). Bar: $10 \mu \mathrm{m}$.

intravacuolar tachyzoites, suggesting that BFA directly targets the GC (Figs. 5e,f). Thus, the infected cultures were treated for $2 \mathrm{~h}$ with $\mathrm{BFA}$ and after that incubated with $\mathrm{C}_{6^{-}}$ NBD-Cer for $5 \mathrm{~h}$. A disperse fluorescence pattern appeared in the cytoplasm, but no fluorescent stain was noted in the intravacuolar space (Figs. 5g,h). These results were consistent with the previous ultrastructural analyses, where incubation with BFA showed vesiculations of cytoplasm organelles, and this alteration resulted in the interruption of transit of lipids to the intravacuolar parasite.

\section{DISCUSSION}

The Golgi complex is an important organelle for cell homeostasis. In T. gondii, the GC is also important in the 
functioning of the secretory complex that is necessary for invasion and development within the host cell. Inside the parasitophorous vacuole space, Toxoplasma also depends on lipid traffic from the GC and other molecules from the cytoplasm of the host cell. However, there is little direct evidence about the interrelation between GC and intravacuolar tachyzoite development in the host cell. Our results suggest that anti-Golgi agents decrease infection, with elimination of intracellular tachyzoites during different lengths of incubation.

The most efficient compound was Monensin. The faster effect of Mon on extra- or intracellular parasites could be related to its ionophore action, with allows exchange of $\mathrm{Na}^{+}$ and $\mathrm{H}^{+}$across biological membranes. This results in an increase in internal $\mathrm{pH}$ and provokes water entry, inducing swelling of all Golgi cisternae and arrest of vesicle secretor transport [14,17]. Thus, Mon was able to interrupt vital parasite secretory organelles, as observed in ultrastructural analyses. The effects of Mon on the parasite were drastic but few toxic effects were observed in the host cell. This aspect may be related to the mechanism of action of Mon in the presence of cholesterol in the membranes. Cholesterol enhances $\mathrm{Na}^{+}$diffusion. In mammalian cells the effect of Mon is greater in trans Golgi-membrane (cholesterol rich) than in cis Golgi-membrane (low in cholesterol), as reviewed in Dinter and Berger [14]. This cholesterol-dependent sensitivity to Mon may explain the higher impact of monensin on intracellular tachyzoites than the host cell.

BFA also has a profound structural effect at different sites in the endomembrane cell system, causing morphological changes in the endoplasmic reticulum, endosomes, lysosomes and Golgi complex and also inhibiting secretory pathways [21,22]. Retinoic acid induces Golgi swelling [18], modifies the endocytic routing in cells and blocks new protein secretion [24]. The effects of BFA and RA on intracellular parasites were observed only after 5 hours of incubation. This may be related to the period of time taken by parasites of strain $\mathrm{RH}$ to accomplish their first cycle multiplication, which is about 6 to 8 hours. Thus, the effect of the drugs within 5 hours suggests that secretion events necessary for parasite development were interrupted. Okadaic acid treatment arrests the endoplasmic reticulum (ER)-Golgi membrane traffic in host cells, inducing a profound reduction in both fluid phase and receptormediated endocytosis as well inhibition of endosome fusion and phosphatase protein activity [25]. Besides this, OA causes fragmentation and disassembly of the Golgi sacks with the formation of tubulovesicular Golgi clusters [25] and abolishes vesicular transport $[14,26,27]$. These extensive effects affected the parasite secretion system and also host cells, where drastic cytotoxic effects were noted.

The effect of Mon on parasites was not reversed when the drug was removed from the infected cultures, unlike BFA or RA, which had reversible effects on parasite multiplication. These results suggest that the perturbation of ion exchange was more efficient than those effects observed with BFA or RA. The efficient and faster Mon anti-parasite action may be related to reduction of the drastic toxic effects on the host cells. In addition, drug associations (Mon+BFA+RA) did not change the effect of Mon by itself, demonstrating the effective action of Mon on parasites.
Ultrastructural studies showed that cytotoxic effects in the presence of OA, Mon, BFA and RA affected Golgi structure and function [14,17-20]. Mon induced complete endomembrane parasite disorganization, and enlargement of PVs containing parasites. BFA caused parasite organelle disorganization; moreover, its effects were also noted in the host cell Golgi complex. However, all these effects were more fatal to intravacuolar parasites. Parasite death following treatment with the two drugs, Mon and BFA, occurred at different points. Both of them had profound structural effects on other organelles such as the endoplasmic reticulum, traffic vesicles, endosomes and lysosomes [21,22]. Connected with this, Ferri and Kroemer [23] showed that the endoplasmic reticulum, lysosomes and the Golgi complex are also major points of integration of proapoptotic signaling or damage sensing. It will be interesting to consider these effects during the death of the parasite in the presence of these drugs. RA led to tachyzoite elimination only after $24 \mathrm{~h}$ of treatment. Wu [24] suggested that RA preferentially modifies the endocytic routing in cells and blocks new protein secretion. This mode of action could be related to the delay in the anti-parasite effect of RA when compared with the other drugs. Although okadaic acid arrested parasite development, drastic toxic effects and death in both parasite and host cells were observed before this. Okadaic acid caused wide fragmentation and detachment in the Golgi sacks with the formation of tubulovesicular Golgi clusters and interruption of vesicular transport [14,25-27]. These cellular effects of OA were fatal to both the host cell and intravacuolar tachyzoites.

As discussed above, the effect of the drugs, mainly Mon and BFA, on the structure and functionality of the Golgi complex, led to interruption of development and the elimination of the intracellular tachyzoites. Some papers have used fluorescent probes to stain the GC and demonstrate its function in lipid traffic from the $\mathrm{GC}$ to the plasma membrane [10-12]. In same way, Melo et al. [9] and other authors [10-12] have demonstrated the relationship between host cell molecule traffic and PVs containing $T$. gondii [9]. Given that the Golgi complex has a central role in metabolite traffic mediated by coated vesicles [6], we analyzed what happened with exogenous lipids $-\mathrm{C}_{6}-\mathrm{NBD}$ ceramide - traffic in infected cells in the presence of Mon and BFA. Several studies showed that C6-NBD-ceramide initially stains the Golgi complex where it is metabolized to the fluorescent products $\mathrm{C}_{6}$-NBD-glycosylceramide and sphingomyelin which are localized on the plasma membrane [16]. Melo et al. [9] demonstrated that intravacuolar parasites take up the fluorescent $\mathrm{C}_{6}$-NBD-Ceramide metabolites - $\mathrm{C}_{6}$-NBD- glycosylceramide and sphingomyelin - after $5 \mathrm{~h}$ of exogenous lipid incubation, as demonstrated in this report. When infected cultures were treated with Mon and incubated with the fluorescent probe, the GC of host cells continued to fluoresce, but intracellular parasites did not incorporate the fluorescent lipid. The inability of intravacuolar parasites to capture fluorescent lipids during incubation with Mon or BFA strongly suggests that the entry of these lipids into the PV is dependent on parasite viability and is not only due to a simple diffusion process. The fact that the GC of host cells remained stained during Mon incubation, but in the presence of BFA the fluorescent 
pattern was diffuse in the cytoplasm of the host cell, is consistent with the ultrastructural results shown earlier.

In summary, the interruption of Golgi complex functions and other organelles in the parasites may induce tachyzoite death. However each anti-Golgi agent used here had different effects on the infection. Those related to the endocytic pathway, such BFA and AR, affected parasites in their period of multiplication, and also harmed the host cells after a longer period of incubation. On the other hand, Mon affected parasites after 1 hour of incubation, including both intra- and extracellular tachyzoites. This means that Mon was more harmful to parasites than host cells. In this context we may consider that infected cells recovered from infection following treatment with Mon. Connected with this, Melo et al. [28] demonstrated that interruption of parasite cell multiplication using hydroxyurea also led to tachyzoite elimination, and as shown by Melo and Carvalho [29], this elimination was associated with lysosome-parasitophorous vacuole fusion, resulting in complete digestion of the parasites. In the present study, we did not perform assays to verify whether the PVs containing parasites after treatment with anti-Golgi agents also interact with the cell digestive pathway; however it is important to consider this possibility. In addition, we suggest that the perturbation of the system by Mon is a promising tool with which to explore fully the weak points of the parasite.

\section{CONCLUSION}

The structural alterations in the Golgi complex and secretory organelles in intracellular tachyzoites interrupted their development, combined with the blocking of lipid transport to intravacuolar parasites, which contributed to collapse and parasite death, mainly in the presence of Monensin. The effect of Mon on the parasite Golgi complex was the principal engine leading to its disorganization and elimination, perhaps due to its ionophore action. The drugs used in this report demonstrated that several targets, mainly in the secretory pathway, are affected more in the intracellular parasite than in the host cell. Intracellular tachyzoite survival and development depends on the host cell Golgi apparatus, as demonstrated here using anti-Golgi drugs.

\section{ACKNOWLEDGEMENTS}

We thank the Fundação de Amparo à Pesquisa do Estado do Rio de Janeiro (FAPERJ) for financial support.

\section{REFERENCES}

[1] Dubremetz. Host cell invasion by Toxoplasma gondii. Trends Microbiol 1998; 6: 27-36.

[2] Pfefferkor ER. Cell biology of Toxoplasma gondii. In: Modern Parasite Biology (Wyler, D.J. Ed.) 1990; 26-50. NY, USA.

[3] Melo EJT, Carvalho TU, De Souza W. Penetration of Toxoplasma gondii into host cells induces changes in the distribution of the mitochondria and the endoplasmic reticulum. Cell Struct Funct 1992; 17: 311-17.

[4] Melo EJT, De Souza W. Relationship between the host cell endoplasmic reticulum and the parasitophorous vacuole containing Toxoplasma gondii. Cell Struct Funct 1997; 22: 317-23.

[5] Sinai AP, Webster P, Joiner KA. Association of host cell mitochondria and endoplasmic reticulum with the Toxoplasma gondii parasitophorous vacuole. J Cell Sci 1997; 110: 2117-28.
[6] Glick BS. Organization of the Golgi apparatus. Curr Opin Cell Biol 2000; 12: 450-56.

[7] Pelletier L, Stern CA, Pypaert M, et al. Golgi biogenesis in Toxoplasma gondii. Nature 2002; 418: 548-52.

[8] Joiner KA, Ross DS. Secretory traffic in the eukaryotic parasite Toxoplasma gondii: Less is more. J Cell Biol 2002; 157: 557-63.

[9] Melo EJT, De Souza W. Pathway of $\mathrm{C}_{6}$-NBD-ceramide on the host cell infected with Toxoplasma gondii. Cell Struct Funct 1996; 21: 47-52.

[10] Coppens I, Sinai AP, Joiner KA. Toxoplasma gondii exploits host low-density lipoprotein receptor-mediated endocytosis for cholesterol acquisition. J Cell Biol 2000; 149: 167-80.

[11] Charron AJ, Sibley LD. Host cells mobilized lipid resources for the intracellular parasite Toxoplasma gondii. J Cell Sci 2002; 115: 3045-59.

[12] Seghal A, Bettiol S, Pypaert M, et al. Peculiarities of host cholesterol transport to the unique intracellular vacuole containing Toxoplasma. Traffic 2005; 6: 1125-41.

[13] Coppens I. Contribution of host lipids to Toxoplasma pathogenesis. Cell Microb 2006; 8(1): 1-9.

[14] Dinter A, Berger EG. Golgi-disturbing agents. Histochem Cell Biol 1998; 109: 571-90.

[15] Melo EJT, Mayerhoffer RO, De Souza W. Hydroxyurea inhibits intracellular Toxoplasma gondii multiplication. FEMS Microbiol Lett 2000; 185: 79-82.

[16] Martin OC, Pagano PE. Internalization and sorting of a fluorescent analogue of glucosylceramide to the Golgi apparatus of human skin fibroblasts: Utilization of endocytic and nonendocytic transport mechanisms. J Cell Biol 1994; 125: 769-81.

[17] Zhang GF, Driouich A, Staehelin AL. Effect of monensin on plant Golgi: Re-examination of the monensin-induced changes in cisternal architecture and functional activities of the Golgi apparatus of sycamore suspension-cultured cells. J Cell Sci 1993; 104: 819-31.

[18] Brown R, Gray RH, Bernstein IA. Retinoids alter the direction of differentiation in primary culture of cutaneous keratinocytes. Differentiation 1985; 28: 268-78.

[19] Hyodoh F. Effects of retinoic acid on the differentiation of THP-1 cells lines containing aneuploid or diploid chromosomes. Cell Struct Funct 1987; 12: 225-42.

[20] Wlodkowic D, Skommer J, Pelkonen J. Brefeldin A triggers apoptosis associated with mitochondrial breach and enhances HA 14-1 and anti-Fas-mediated cell killing in follicular lymphoma cells. Leuk Res 2007; 31: 1687-1700.

[21] Lippincott-Schwartz J, Yuan L, Tipper C, Amherdt M, Orci L, Klausner RD. Brefeldin A's effects on endosomes, lysosomes, and the TGN suggest a general mechanism for regulating organelle structure and membrane traffic. Cell 1991; 67(3): 601-16.

Pelham HR. Multiple targets of brefeldin A. Cell 1991; 67: 449-51.

Ferri KC, Kroemer G. Organelle-specific initiation of cell death pathway. Nat Cell Biol 2001; 2: E255-E263.

Wu YN, Gadina M, Tao-Cheng JH, Youle RJ. Retinoic acid disrupts the Golgi apparatus and increases the cytosolic routing of specific protein toxins. J Cell Biol 1994; 125: 743-53.

[25] Lucocq J, Warren G, Pryde J. Okadaic acid induces Golgi apparatus fragmentation and arrest of intracellular transport. J Cell Sci 1991; 100: 753-59.

[26] Thyberg J, Moskalewski S. Disorganization of the Golgi complex and the cytoplasmic microtubule system in $\mathrm{CHO}$ cells exposed to okadaic acid. J Cell Sci 1992; 103: 1167-75

[27] Hoe MH, Slusarewicz P, Misteli T, Watson R, Warren G. Evidence of recycling of the resident medial/trans Golgi enzyme, $\mathrm{N}$ acetylglucosaminyltransferase I, in ldlD cells. J Biol Chem 1995; 270: 25057-63.

[28] Beiral H, Melo, EJT. Effect of hydroxyurea on the intracellular multiplication of Toxoplasma gondii, Leishmania amazonensis and Trypanosoma cruzi. Braz J Med Biol Res 2003; 36(1): 65-69.

[29] Carvalho CS, Melo EJT. Acidification of the parasitophorous vacuole containing Toxoplasma gondii in the presence of hydroxyurea. An Acad Bras Cienc 2006; 78(3): 475-484. 
[30] Pagano RE, Sepanski MA, Martin OC. Molecular trapping of a fluorescent ceramide analogue at endogenous lipids provides a
trans-Golgi marker for both light and electron microscopy. Cell Biol 1989; 109: 2067-79.

Received: August 20, 2008

Revised: September 26, 2008

Accepted: December 09, 2008

(C) Tharkar and Viswanathan; Licensee Bentham Open.

This is an open access article licensed under the terms of the Creative Commons Attribution Non-Commercial License (http: //creativecommons.org/licenses/bync/3.0/), which permits unrestricted, non-commercial use, distribution and reproduction in any medium, provided the work is properly cited. 